\title{
O Acompanhamento Terapêutico como Estratégia de Cuidado na Atenção Psicossocial
}

Therapeutic Follow-Up As A Strategy For Attention In The Psychosocial Care

El Acompañamiento Terapéutico Como Estrategia De Cuidado En La Atención Psicosocial

Manoel de Lima Acioli

Neto \& Paulo Duarte de

Carvalho Amarante

Universidade Federal de Pernambuco
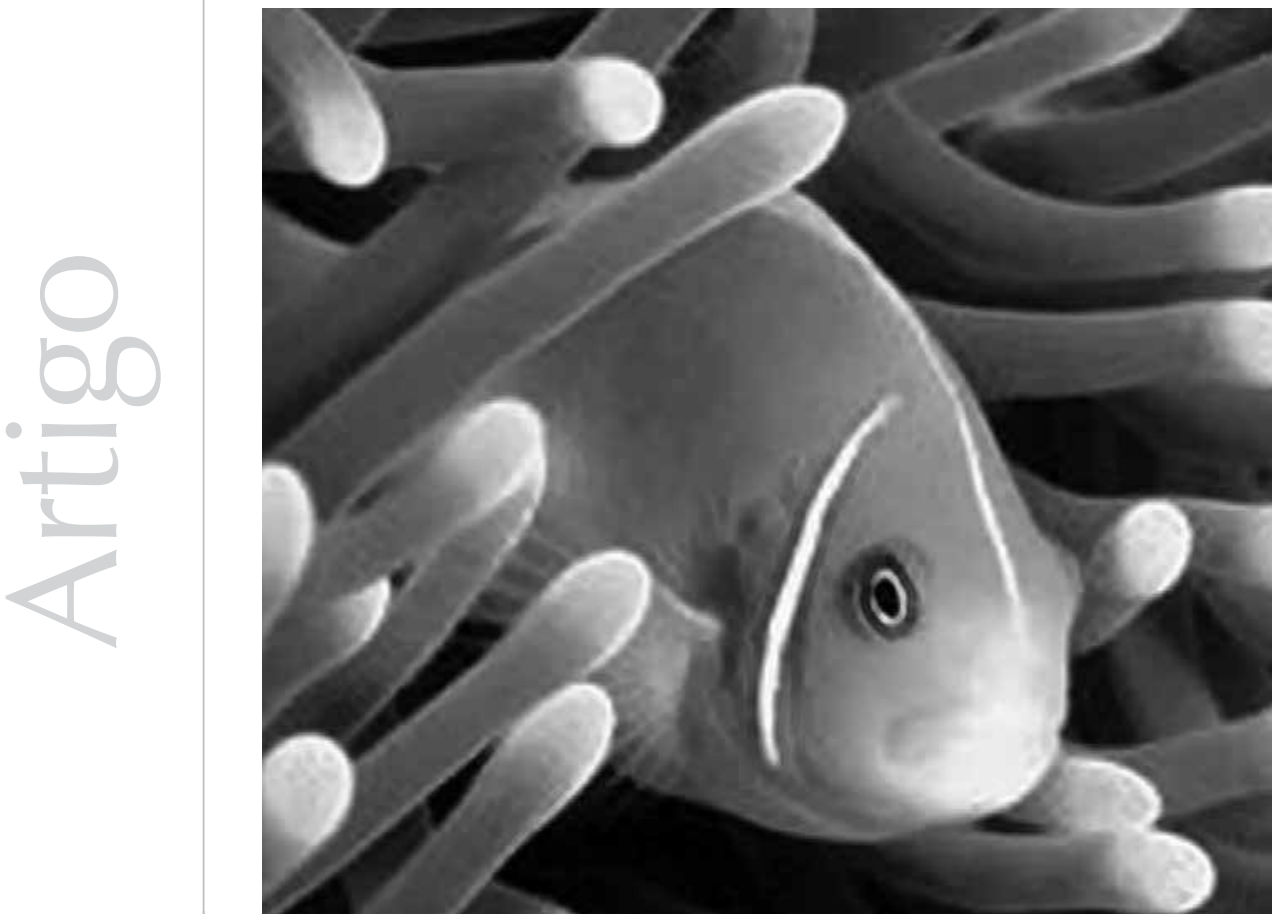
Resumo: $\mathrm{O}$ acompanhamento terapêutico constitui um importante instrumento de integração de projetos assistenciais centrados na atenção psicossocial. Suas ações se inserem como práticas opostas aos modelos asilares de tratamento, alinhando-se às propostas da reforma psiquiátrica e sanitária. Diante disso, questionase sobre a forma como a atenção psicossocial vem sendo desenvolvida dentro dos Centros de Atenção Psicossocial e buscam-se alternativas para a superação dos impasses da rede de saúde mental. O objetivo deste trabalho é refletir sobre as possibilidades de contribuição do acompanhante terapêutico dentro da atenção psicossocial. Assim, pode-se considerar que a atenção psicossocial ainda está em processo de construção como base assistencial dos atuais equipamentos da reforma psiquiátrica. Com isso, um ator que poderia atuar circulando nesses diferentes setores, nesses diferentes espaços, é o acompanhante terapêutico, promovendo a facilitação, a inserção e a construção conjunta do usuário ao seu universo cotidiano e em novas redes culturais/interacionais.

Palavras-chave: Acompanhamento terapêutico. Centro de atenção psicossocial - (CAPS). Saúde mental. Serviços de saúde mental.

\begin{abstract}
The therapeutic follow-up is an important tool for integrating service projects focused on psychosocial care. These actions are work practices as opposed to models of asylums treatment, aligning the proposed reform and psychiatric health. Therefore, it is uncertain how psychosocial care has been developed within the Psychosocial Care Centers, and alternatives to overcome the impasses of mental health network are sought. The objective of this paper is to discuss the possibility of the therapist contribution in psychosocial care. Thus, one can consider that psychosocial care is still in the building process as the basis of the current healthcare equipment of psychiatric reform. Thus, an actor who could act circulating in these different sectors, these different spaces is the therapist, promoting the user's facilitation, integration and joint construction in his/her everyday world and in new cultural /interactional networks.
\end{abstract}

Keywords: Therapeutic follow-up. Psychosocial Atenttion Center - CAPS. Mental health. Mental health services.

Resumen: El acompañamiento terapéutico constituye un importante instrumento de integración de proyectos asistenciales centrados en la atención psicosocial. Sus acciones se insieren como prácticas opuestas a los modelos asilares de tratamiento, alineándose a las propuestas de la reforma psiquiátrica y sanitaria. Ante eso, se cuestiona sobre la forma como la atención psicosocial viene siendo desarrollada dentro de los Centros de Atención Psicosocial y se buscan alternativas para la superación de los impases de la red de salud mental. El objetivo de este trabajo es reflexionar sobre las posibilidades de contribución del acompañamiento terapéutico dentro de la atención psicosocial. Así, se puede considerar que la atención psicosocial aún está en proceso de construcción como base asistencial de los actuales equipos de la reforma psiquiátrica. Con eso, un actor que podría actuar circulando en esos diferentes sectores, en esos diferentes espacios, es el acompañamiento terapéutico, promoviendo la facilitación, la inserción y la construcción conjunta del usuario a su universo cotidiano y en nuevas redes culturales/interaccionales.

Palabras clave: Acompañamiento terapéutico. Centro de Atención Psicosocial - CAPS. Salud mental. Servicios de salud mental.

O acompanhamento terapêutico (AT) constitui um importante instrumento de integração de projetos assistenciais centrados na atenção psicossocial. Suas ações se inserem como práticas opostas aos modelos asilares de tratamento, alinhando-se a propostas da reforma psiquiátrica e sanitária (Fiorati, 2006).

A atenção psicossocial se apresenta como projeto ético-estético-político, no sentido proposto por Guattari (1996), que busca a autonomia do indivíduo a partir do desenvolvimento de sua cidadania e participação política e integração ao território, além da horizontalização das relações profissionais intra e interinstitucionais (Brasil, 1988, 1994, 2002, 2010); assim, vem consolidando-se como o referencial teórico predominante do modelo assistencial em saúde mental no Brasil, constituindo as bases da política pública oficial (Guljor, 2003). Desse modo, a atenção psicossocial caracteriza-se como uma diretriz da reforma psiquiátrica brasileira e assume um papel preponderante no campo assistencial por nortear a construção de novas práticas e serviços em saúde mental. 
Essa construção advém de movimentos reformistas em torno do cuidado à loucura, sendo ancorada, principalmente, no modelo italiano da psiquiatria democrática, o qual tinha como pressupostos uma reorganização dos serviços em torno de práticas de territorialização (e desinstitucionalização), atribuição de responsabilidade ao louco e questionamento do poder psiquiátrico em torno das ações de saúde mental. Oficialmente, a inserção do modelo de atenção psicossocial se inicia com a implantação do Centro de Atenção Psicossocial Luiz Cerqueira e dos Núcleos de Atenção Psicossocial, na cidade de Santos (SP) - década de 80 (Amarante, 2000).

Busca-se, portanto, que a atenção à loucura seja promovida fora do âmbito asilar (que a segregou e tutelou por tantas décadas), através da integração de diversos setores sociais, em especial a saúde, a assistência social, a educação e a Justiça (Brasil, 2002). Contudo, essas ações ainda se encontram delimitadas em torno de alguns serviços, sendo questionável a existência de uma rede integrada em saúde mental.

De acordo com autores como Costa-Rosa, Luzio e Yasui (2001), a atenção psicossocial pode ser descrita a partir da seguinte matriz: 1) a integralidade das ações de cuidado no território, 2) horizontalização das relações profissionais e intrainsitucionais, 3) a construção de uma ética da autonomia e da singularização do indivíduo em sofrimento psíquico e 4) a implicação subjetiva desse indivíduo no cuidado territorializado. Além disso, de acordo com a III e a IV Conferência Nacional de Saúde Mental, enfatiza-se que a atenção psicossocial deve possuir como premissas: 5) a intersetorialidade nas práticas desenvolvidas e 6) a desinstitucionalização do usuário aos serviços (Brasil, 2010, 2002).
Nesse sentido, o trabalho desenvolvido pelo acompanhante terapêutico se alinha a essa lógica. O AT pode ser definido como uma prática cujo espaço clínico está nas ruas (Bezerra \& Dimenstein, 2009; Palombini, 2009), como um dispositivo clínico que almeja, entre outros, aproximar o sujeito das ofertas de laço social (Pitiá \& Furegato, 2009; Hermann, 2010; Reis-Neto, Pinto, \& Oliveira, 2011), resgatando vínculos, sua cidadania e sua circulação em espaços que façam sentido para o portador (Carniel \& Pedrão, 2010; Chauí-Berlinck, 2010).

De acordo com Fiorati e Saeki (2008), essas ações de inserção social promovem a expressão de formas particulares de existência. Para Silva, Costa e Neves (2010), através dessas estratégias, desenvolve-se um recurso em saúde mental alternativo à internação em hospital psiquiátrico. Estellita-Lins, Oliveira e Coutinho (2009) consideram que o AT se caracteriza como uma intervenção em saúde mental baseada em cuidados domiciliares, facilitando um retorno a condições existenciais perdidas e, por conseguinte, ambicionadas. Pitiá e Furegato (2009) descrevem a ação do AT como um instrumento de ação terapêutica em que se considera o sujeito em seu contexto socio-histórico-psíquico-biológico e cultural.

Com origem na década de 60, na Argentina, o trabalho do acompanhante terapêutico supre uma necessidade clínica para pessoas que não tinham respostas para as ações terapêuticas tradicionais (Mauer \& Resnizky, 1987; Sereno \& Porto, 1991). No Brasil, com o surgimento das primeiras comunidades terapêuticas, esse profissional surge com a denominação de auxiliar psiquiátrico (Ibrahim, 1991).

O trabalho do auxiliar psiquiátrico era desenvolvido, nessa época, dentro das 
De acordo com a Portaria $n^{\circ} 336 / 02$, do Ministério da Saúde, o CAPS assume o papel de regulador da porta de entrada na saúde mental e de coordenador do cuidado na rede ao indivíduo com transtorno mental severo e persistente (Brasil, 2002). instituições, sendo suas funções ligadas ao desenvolvimento de jogos e festas, dentre outras atividades recreacionais. Contudo, na década de 70, com a política do regime militar de internação asilar em detrimento de outros modelos de cuidado, as comunidades terapêuticas entram em declínio e os auxiliares psiquiátricos perdem seu espaço de atuação. Por conseguinte, começam a ser solicitados para trabalhos particulares, dentro do ambiente doméstico, que proporcionam o contato direto com o cotidiano e o universo familiar do indivíduo em sofrimento psíquico (Berger, Morettin \& Braga Neto, 1991).

Com isso, esses profissionais passaram a ser solicitados para trabalhos particulares na residência dos pacientes e os acompanhamentos passaram a ser feitos no ambiente doméstico, o que proporcionava a oportunidade de entrar em contato direto com seu cotidiano e universo familiar. Esse modelo interventivo definiu sua área de atuação, principalmente com pacientes psicóticos, em uma busca por reintegrá-los na sociedade e para reconstruir os vínculos familiares (Zamignani \& Wielenska, 1999). Apesar de ter se destacado, inicialmente, no acompanhamento de casos de psicose, esse profissional se consolida na assistência de diversos transtornos.

Desse modo, o acompanhante terapêutico poderia ser situado, no contexto da reforma psiquiátrica, como um agente que permite novas formas de sociabilidade apoiadas na interação dialógica, desenvolvendo posicionamentos que saem da interdição para assumirem a interlocução, o livre trânsito e para reposicionar os sujeitos dentro da dimensão subjetiva, sociocultural e histórica. Essas ações tornam o sujeito agente produtor e transformador, mobilizando-o como protagonista do seu tratamento (Fiorati \&
Saeki, 2008). Assim, esse trabalho possui uma especificidade que possibilita um fortalecimento das estratégias desenvolvidas pela saúde mental para a implantação da atenção psicossocial dentro das práticas de cuidado ofertadas pela rede, a qual encontra como dispositivo por excelência os Centros de Atenção Psicossocial.

Nesse sentido, questiona-se sobre a forma como a atenção psicossocial vem sendo desenvolvida dentro dos Centros de Atenção Psicossocial (CAPS), equipamentos que têm como finalidade a implantação e o funcionamento dessa estratégia de atenção no âmbito da saúde, buscando-se alternativas para a superação dos impasses da rede de saúde mental. Assim, o objetivo deste trabalho é refletir sobre as possibilidades de contribuição do acompanhante terapêutico dentro da atenção psicossocial.

\section{Os centros de atenção psicossocial e novas cronicidades}

De acordo com a Portaria no 336/02, do Ministério da Saúde, o CAPS assume o papel de regulador da porta de entrada na saúde mental e de coordenador do cuidado na rede ao indivíduo com transtorno mental severo e persistente (Brasil, 2002). Sua proposta terapêutica se adapta a um modelo de não internação, promovendo o cuidado de atenção diária sem retirar o usuário de seu território. O ministério da Saúde estabelece que os CAPS devem prestar como assistência ao usuário de seus serviços: atendimento individual e grupal, atendimento por oficinas terapêuticas, visitas domiciliares, atendimento à família e atividades comunitárias que insiram o usuário em seu meio social, comunitário e familiar (Brasil, 2002). 
Desse modo, os CAPS propõem desenvolver um projeto terapêutico individualizado que tenha como objetivos o tratamento e a reabilitação psicossocial, e o trabalho deve estar em consonância com questões de ordem social presentes no cotidiano dos usuários. Por isso, esse projeto deve desenvolver-se inserido em uma clínica ampliada e centrada no sujeito (OnockoCampo \& Furtado, 2006); além disso, deve alinhar-se aos princípios do Sistema Único de Saúde (SUS), buscando garantir acesso, integralidade e resolutividade na atenção prestada. Diante disso, questiona-se: o CAPS tem funcionado dessa forma?

Apesar de sua proposta assistencial, ainda predomina a reprodução de modelos biomédicos dentro dos CAPS e a desresponsabilização pelo usuário dentro da rede (Dimenstein et al., 2009; Pegoraro \& Caldana, 2008; Venâncio, 2007). Com isso, o cuidado se torna fragmentado, havendo apenas uma relação pontual entre profissional/usuário, reafirmando a lógica ambulatorial que se propõe ultrapassar (Dimenstein et al., 2009). Essa lógica remete à produção de novas cronicidades aos usuários, o que revela que ainda não se rompeu totalmente com o modelo manicomial. Como aponta Amarante, apesar dos incontestáveis avanços conquistados, nós nos situamos diante de um cenário em que os objetivos eticamente orientados para a abolição dos tratamentos de cunho manicomial estão longe de serem alcançados em sua plenitude.

Dimenstein et al. ainda demonstram que, no funcionamento de muitos CAPS, observase um modo de funcionamento centrado no trabalho individualizado de diferentes técnicos, com pouca inserção no território. Os autores também destacam que, em alguns casos, as atividades e as oficinas se tornam tarefas rotineiras em que não se permite a criação de novas estratégias de cuidado, tornando-se dispositivos de ocupação do tempo.

Um fenômeno observado por alguns autores como uma decorrência desse modo de funcionamento é a institucionalização do usuário produzida nos CAPS. Esse é um tema polêmico e que remete à produção de novas cronicidades, expressas na retenção de usuários, em modos de gestão resistentes em operar fora dos serviços e nas portas de saída e circulação na rede (Alverga \& Dimenstein, 2006). De acordo com Barros (2003), existem três "ordens de cronicidade":

a) as novas cronicidades dos pacientes que se tornam usuários-pacientes, pois estão e/ ou são postos de forma passiva frente ao desafio de produzir outra subjetivação. Essa produção decorre de fatores como o contexto sociofamiliar (refratário à inclusão) e sociopolítico (adverso e precário). Além disso, podem ser apontadas as condições socioculturais marcadamente discriminatórias advindas do estigma dos pacientes psiquiátricos;

b) a cronicidade dos modos de gestão, dos dispositivos e dos profissionais, que se reflete na dificuldade em conciliar a discussão clínica com a análise dos processos trabalhistas e institucionais. Essa cronicidade também é observada na dificuldade de a formação permanente ser aliada às práticas dos serviços, de modo que as marcas da segmentarização, dos especialismos e da centralidade (não territorialização das práticas nos contextos diversos) da formação sejam superadas;

c) a cronicidade que se produz devido à inexistência ou à fragilidade de uma efetiva 
rede de atenção em saúde e, em especial, em saúde mental, que se verifica na existência desorganizada de várias portas de entrada e na falta de portas de saída.

Dessa forma, desinstitucionalizar é ultrapassar fronteiras sanitárias, é enfrentar o desafio da intersetorialidade e do trabalho em rede, o que implica a adoção de modelos de atenção integral de base territorial (Liberato, 2009). Como afirma Pitta (2001), a assistência em saúde mental propõe a descentralização da atenção, através da interdisciplinaridade e da intersetorialidade, vinculando o conceito de saúde mental aos conceitos de cidadania e de produção de vida. Esse processo destinase a construir o melhor nível possível de autonomia do indivíduo, no exercício de suas funções sociais (Pitta, 2001).

Contudo, embora se preconize a promoção desse cuidado descentralizado, territorializado, o usuário recebe prioritariamente assistência dentro dos serviços, transformando, muitas vezes, o território em adscrição geográfica, em vez de espaços de convivência, nos quais ocorrem a significação e a construção da familiaridade e do pertencimento a esse local. Desse modo, o CAPS, na forma como tem funcionado em muitos serviços, mostra-se um dispositivo que encontra dificuldades em oferecer o cuidado proposto pela atenção psicossocial, sendo observada a necessidade de construção de novos dispositivos para lidar com as questões de saúde mental. Essa dificuldade decorre dos percalços enfrentados no processo de articulação com outros equipamentos, decadentes pela precariedade de recursos físicos, humanos e materiais e de condições limitadas para garantir a assistência integral de pessoas com transtornos mentais graves e persistentes. Amarante ressalta que o processo de desinstitucionalização se faz a partir da trilogia: negação, superação e invenção. Como destaca, ainda:

Mais que o concreto, as lajes, as grades
do hospício, é esse conjunto que
sobredetermina gastos, olhares, posturas
e exercita limites, intolerância, e diferenças
presentes no próprio hospício, mas
presentes também no ambulatório, centros
de saúde mental comunitários, hospital-
dia, enfermarias psiquiátricas em hospitais
gerais, locais de trabalho, salas de aula,
meio familiar, entre outros. (Amarante,
1994, p. 115)

A transformação das políticas psiquiátricas produziu o desenvolvimento de novos serviços, situação social que revolucionou práticas sociais forjadas há longo tempo. Com isso, o sujeito, relegado às margens da sociedade, volta ao seu centro. Mas, e os preconceitos que justificavam seu confinamento? O desenvolvimento de novas práticas demolirá tão facilmente as barreiras simbólicas como as materiais? (Jodelet, 2005).

Desse modo, torna-se imprescindível que esse processo não fique somente no desenvolvimento de serviços substitutivos, mas que ocorra mudança no paradigma das ações em saúde mental, através da transformação das representações partilhadas socialmente pela loucura (Amarante, 2000, 2007).

Em consonância com essas questões, autores como Yasui e Costa-Rosa (2008) concebem que o cuidado ofertado pela atenção psicossocial deve perpassar a lógica do centro de atendimento, desenvolvendo uma rede de assistência que possa garantir a assistência do usuário em suas mais diferentes esferas, sendo a equipe seu principal alicerce, e o território, seu espaço de atuação. O CAPS pode ser considerado um centro de referência, mas não o único lócus do cuidado. 
Assim, dois dispositivos demonstrados que têm possibilidade de solucionar esse problema são os Núcleos de Apoio à Saúde da Família (NASF) e o Matriciamento (Yasui \& Costa-Rosa, 2008; Dimenstein et al., 2009), que garantem ações integralizadas ao cuidado do indivíduo em sofrimento psíquico. Esses dispositivos viabilizam o suporte técnico às equipes de ações básicas de saúde, assim como intervenções e atendimentos conjuntos, promovendo corresponsabilização pelos casos. Com isso, ocorre melhor articulação entre as equipes dos CAPS com a atenção básica, deslocando o cuidado dos espaços tradicionalmente institucionalizados para um trabalho com as famílias e as comunidades. Mas surge ainda o questionamento: será possível alcançar ações integrais aos usuários com esses dispositivos?

A integralidade pode ser alcançada através da integração de serviços por meio de redes assistenciais, reconhecendo a interdependência dos atores e organizações, sendo, portanto, indispensável desenvolver mecanismos de cooperação e de coordenação próprios de uma gestão eficiente e responsável dos recursos coletivos, a qual responda às necessidades de saúde individuais em âmbitos local e regional (Hartz \& Contandriopoulos, 2004).

Entretanto, um componente indissociável da integralidade é a intersetorialidade (Alves, 2001), pois a complexidade da realidade social exige um olhar que não se esgota no âmbito de uma única política social (Junqueira, 2004). Para intervir nos problemas sociais, torna-se necessário que os diversos atores sociais e organizacionais participem da gestão das políticas sociais, privilegiando a ação intersetorial (Dabas, 1995).

Assim sendo, como afirma Liberato:

\begin{abstract}
Se a constituição de uma rede de serviços substitutivos integrada entre si e com outros equipamentos sociais presentes nas comunidades é algo imprescindível para o avanço da reforma, esse é um dos aspectos que apresentam mais fragilidades, pois, de fato, ainda não dispomos de uma rede ágil, flexível, resolutiva, onde o trânsito dos usuários é facilitado e o mesmo é acolhido em suas diferentes demandas. Identificamos muito mais serviços isolados, que não se comunicam, fechados em suas rotinas. $(2009$, p. 5).
\end{abstract}

Assim, deve-se apontar como finalidade de uma ação intersetorial a promoção da territorialização do cuidado, fora de estabelecimentos. O território é definido pela significação/ressignificação de um espaço por um determinado grupo social (Faria \& Bortolozzi, 2009), através da produção de familiaridade e propriedade (pertencimento) (Barros, 2006). Com isso, territorialização significa ação culturalmente embasada nas singularidades de cada sujeito em seu universo interacional.

Diante desses problemas, percebese a importância de um cuidado mais individualizado, com um direcionamento para a possibilidade de reconstrução de aspectos relevantes na produção social da vida, demonstrando outras formas de assistência e de inserção social e que evitassem o circuito vicioso de práticas asilares e de novas cronicidades (Fiorati, 2006).

Dessa forma, o acompanhamento terapêutico surge como uma proposta que pode responder a algumas dessas dificuldades. A partir de ações de resgate da cidadania, por meio da vivência partilhada das práticas cotidianas, o AT constrói formas de cuidado que se apoiam na singularização do sujeito. Essas ações envolvem o indivíduo em sua dimensão subjetiva, sem demarcações de territórios 
A partir de ações de resgate da cidadania, por meio da vivência partilhada das práticas cotidianas, o AT constrói formas de cuidado que se apoiam na singularização do sujeito. Essas ações envolvem o indivíduo em sua dimensão subjetiva, sem demarcações de territórios e fragmentação dos saberes e das práticas (Fiorati, 2006). e fragmentação dos saberes e das práticas (Fiorati, 2006). Assim sendo, considerando as premissas da atenção psicossocial em relação à promoção da saúde e sua construção no território do sujeito, considera-se que o AT tenha um papel determinante nesse processo. Com suas ações, os sujeitos podem circular em seus espaços cotidianos, ressignificando suas ações rotineiras, assim como nos benefícios ou danos que podem estar aí atribuídos. O AT se apresenta como um ator que leva o cuidado ao sujeito fora dos muros institucionais, atuando no percurso de suas vidas, em sua relação com o mundo. O seu trabalho constitui uma ação necessária que pode articular a atividade dos CAPS, a atenção psicossocial para o contexto cotidiano de seus usuários, amenizando, inclusive, possíveis efeitos de novas cronicizações. Sua práxis ocorre no encontro com o sujeito em seu dia a dia, em suas atividades rotineiras, em suas atividades de lazer, em suas vivências, e tem como finalidade a promoção de um cuidado fora do espaço institucional, desvinculando a prática da saúde desses espaços.

Entretanto, vale ressaltar, não necessariamente o dispositivo clínico do acompanhamento terapêutico pode ser considerado como o determinante desse processo de construção de uma atenção psicossocial. A forma como se pratica esse dispositivo pode também remeter à quebra de vínculos, promovendo uma atenção tutelada e segregada, com marcas do modelo asilar. O trabalho do AT pode ser estratégico para a atenção psicossocial a partir do momento em que se trabalha dentro dessa lógica assistencial.

\section{Considerações finais}

Com isso, pode-se considerar que a atenção psicossocial ainda esteja em processo de construção, como base assistencial dos atuais equipamentos da reforma psiquiátrica. Como foi discutido, alguns CAPS, espaços privilegiados de implantação desse projeto, ainda se ancoram em práticas hospitalares/ asilares, produzindo inclusive novas cronicidades para seus usuários (Dimenstein et al., 2009). Além disso, os novos dispositivos desenvolvidos para a adequação desse cuidado promovem melhoria na rede, mas ainda não contemplam a intersetorialidade, embora consigam inserir um cuidado de base territorial.

Nesse sentido, parte-se de uma concepção de cuidado que implica a atuação dentro do cotidiano, dos espaços de convivência dos usuários. Por isso, torna-se importante a criação de novos circuitos de socialização para que se construa uma rede que funcione com base na participação social em diversas áreas, em espaços de inserção. Assim, são necessários equipamentos intersetoriais: centros de arte, bibliotecas, cinemas, museus, espaços com apresentações artísticas, assim como escolas de artes e ginásios de esporte, dentre outros, espaços em que diferentes setores possam contribuir para o cuidado e o desenvolvimento da autonomia do indivíduo em sofrimento psíquico.

Nesse sentido, considera-se que um ator que poderia atuar circulando nesses diferentes setores, nesses diferentes espaços, é o acompanhante terapêutico, promovendo a facilitação, a integração e a construção conjunta do usuário ao seu universo cotidiano e a novas redes culturais/interacionais. Reinserir é construir possibilidades, entrando nos universos da vida cotidiana através de vivências e de práticas partilhadas pela sociedade, não por meio de práticas alternativas, excludentes, voltadas unicamente para pessoas com problemas similares. 
Por isso, o trabalho do AT permite o fortalecimento de uma rede intersetorial, na qual a atenção ofertada nos CAPS, por exemplo, pode articular-se com as ações da atenção primária, pois o cuidado promovido por esse ator desenvolve-se em uma elaboração com o usuário, dentro de sua rede comunitária. Assim, o cuidado se torna culturalmente localizado dentro da realidade do sujeito, em conformidade com suas tradições e com os objetivos de sua inserção territorial e autonomia política.

Deve ser ressaltado que é indubitável o avanço promovido pelos atuais modelos de cuidado em saúde mental na atenção à loucura. Contudo, o processo de construção desse cuidado requer constante revisão e atualização. Por isso, diante dessas lacunas e aberturas, destaca-se a necessidade de uma nova estratégia de composição dessa rede de assistência: o acompanhante terapêutico. Esse profissional pode atuar como um dispositivo estratégico aos objetivos da atenção psicossocial de inclusão do usuário e de ressignificação de suas ações dentro de seus espaços de convivência, com desenvolvimento de autonomia e de implicação subjetiva. 
Manoel de Lima Acioli Neto Mestrando do Programa de Pós-Graduação em Psicologia da Universidade Federal de Pernambuco, Recife PE - Brasil.

E-mail: mdlacioli@gmail.com

\section{Paulo Duarte de Carvalho Amarante}

Doutor em Saúde Pública pela Fundação Oswaldo Cruz e docente da Fundação Oswaldo Cruz, Rio de Janeiro - RJ - Brasil.

E-mail: pauloamarante@gmail.com

\section{Endereço para envio de correspondência:}

Rua Azeredo Coutinho, 120, B.8, ap. 201, Várzea. CEP: 50741-110. Recife, PE.

Recebido 18/12/2012, 1a Reformulação 08/05/2013, Aprovado 29/08/2013. 
Alverga, A. R., \& Dimenstein, M. (2006). A reforma psiquiátrica e os desafios na desinstitucionalização da loucura. Interface Comunicação, Saúde, Educação, 10 (20), 299-316. doi: http:// dx.doi.org/10.1590/S1414-32832006000200003

Alves, D. S. (2001). Integralidade nas políticas de saúde mental. In R. Pinheiro \& R. A. Mattos. Os sentidos da integralidade na atenção e no cuidado à saúde (pp.167-176). Rio de Janeiro: IMS ABRASCO.

Amarante, P. (1994). Psiquiatria social e reforma psiquiátrica. Rio de Janeiro: Fiocruz.

Amarante, P. (2000). Loucos pela vida (2a ed.). Rio de Janeiro: SDE/ENSP.

Amarante, P. (2007). Saúde mental e atenção psicossocial. Rio de Janeiro: Fiocruz.

Barros, J. A. (2006). História, espaço e tempo: interações necessárias. Varia História, 22(36), 460-476.

Brasil. Ministério da Saúde. (1988). Relatório Final da I Conferência Nacional de Saúde Mental. Brasília, DF: Centro de Documentação do Ministério da Saúde.

Brasil. Ministério da Saúde. (1994). Relatório Final da II Conferência Nacional de Saúde Mental. Brasília, DF: Centro de Documentação do Ministério da Saúde.

Brasil. Ministério da Saúde. (2002). Relatório Final da III Conferência Nacional de Saúde Mental. Brasília, DF: Centro de Documentação do Ministério da Saúde.

Brasil. Ministério da Saúde. Secretaria de Atenção à Saúde. DAPE. Coordenação Geral de Saúde Mental. (2005). Reforma psiquiátrica e política de saúde mental no Brasil. Brasília, DF:
OPAS. (Documento apresentado à Conferência Regional de Reforma dos Serviços de Saúde Mental: 15 anos depois de Caracas).

Brasil. Ministério da Saúde. (2010). Relatório Final da IV Conferência Nacional de Saúde Mental - Intersetorial. Brasília, DF: Centro de Documentação do Ministério da Saúde.

Berger, E., Morettin, A.V., \& Braga Neto, L. (1991). História. In N. L. Carrozzo (Org.), A rua como espaço clínico (pp. 17-22). São Paulo: Escuta.

Bezerra, C. G., \& Dimenstein, M. (2009). Acompanhamento terapêutico na proposta de alta-assistida implementada em hospital psiquiátrico: relato de uma experiência. Psicol. Clin., 21(1), 15-32. doi: http://dx.doi.org/10.1590/S010356652009000100002

Carniel, A. D., \& Pedrão, L. J. (2010). Contribuições do acompanhamento terapêutico na assistência ao portador de transtorno mental. Rev. Eletr. Enf. (internet), 12(1), 63-72. Recuperado em 08 dezembro, 2012, de http://www.fen.ufg. br/revista/v12/n1/v12n1a08.htm

Chauí-Berlinck, L. (2010). O acompanhamento terapêutico e a formação do psicólogo: por uma saúde humanizada. Arquivos Brasileiros de Psicologia, 62(1), 90-96.

Costa-Rosa, A., Luzio, C., \& Yasui, S. (2001). As conferências de saúde mental e as premissas do modo psicossocial. Saúde em Debate, 25(58), 12-25.

Dabas, E. (1995). De la desestructuración de lo macro a la estructuración de lo micro: las redes sociales en la reconstrución de la sociedad civil. In E., Dabas \& D. Najmanovich (Org.), Redes el lenguaje de los vínculos: Hacia la reconstrucción y el fortalecimiento de la sociedad civil. Buenos Aires: Paidós. 
Referências
Dimenstein, M., Severo, A., Brito, M., Pimenta, A., Medeiros, V., \& Bezerra, E. (2009). O apoio matricial em Unidades de Saúde da Família: experimentando inovações em saúde mental. Saúde e sociedade, 18(1), 63-74. doi: http://dx.doi. org/10.1590/S0104-12902009000100007

Estellita-lins, C., Oliveira, V., \& Coutinho, M. (2009). Clínica ampliada em saúde mental: cuidar e suposição de saber no acompanhamento terapêutico. Ciência \& Saúde Coletiva, 14(1), 195-204. doi: http://dx.doi.org/10.1590/S141381232009000100026

Faria, R. M., \& Bortolozzi, A. (2009). Espaço, território e saúde: contribuições de Milton Santos para o tema da geografia da saúde no brasil. Espaço Geográfico em Análise, 17, 31-41.

Fiorati, R. C. (2006). Acompanhamento terapêutico: uma estratégia terapêutica em uma unidade de internação psiquiátrica. (Dissertação de Mestrado). Escola de Enfermagem de Ribeirão Preto, Universidade de São Paulo, Ribeirão Preto, SP

Fiorati, R. C., \& Saeki, T. (2008). O acompanhamento terapêutico na internação hospitalar: inclusão social, resgate de cidadania e respeito à singularidade. Interface - Comunicação, Saúde, Educação, 12(27), 763-772. doi: http://dx.doi.org/10.1590/ S1414-32832008000400007

Guattari, F. (1996). O novo paradigma estético. In D. F. Schnitman (Org.), Novos paradigmas, cultura e subjetividade (pp. 121133). Porto Alegre: Artes Médicas.

Guljor, A. P. F. (2003). Os centros de atenção psicossocial: um estudo sobre a transformação do modelo assistencial em saúde mental. (Dissertação de Mestrado). Escola Nacional de Saúde Pública da Fundação Oswaldo Cruz, Rio de Janeiro, RJ.

Hartz, Z. M. A., \& Contandriopoulos A. P. (2004). Integralidade da atenção e integração de serviços de saúde: desafios para avaliar a implantação de um "sistema sem muros". Cadernos de Saúde Pública, 20(2), 331-336. doi: http://dx.doi. org/10.1590/S0102-311X2004000800026

Hermann, M. C. (2010). Acompanhamento terapêutico, sua criação em uma montagem institucional de tratamento e as ofertas de laço social. Estilos da clínica, 15(1), 4059. Recuperado em 08 dezembro, 2012, de http:// www.revistasusp.sibi.usp.br/scielo.php?script $=$ sci arttext\&pid $=$ S1415-71282010000100004\&lng=pt\&nrm $=$ iso

Ibrahim, C. (1991). Do louco à loucura: o percurso do auxiliar psiquiátrico no Rio de Janeiro. In N. L. Carrozzo (Org.), A rua como espaço clínico (pp. 43-49). São Paulo: Escuta.

Jodelet, D. (2005). Loucuras e representações sociais. Petrópolis, RJ: Vozes.

Junqueira, L. (2004). Gestão intersetorial das políticas sociais e o terceiro setor. Saúde e Sociedade, 13(1), 25-36. doi: http:// dx.doi.org/10.1590/S0104-12902004000100004

Liberato, M. D. M. (2009). Desinstitucionalizar é ultrapassar fronteiras sanitárias: o desafio da intersetorialidade e do trabalho em rede. Caderno Brasileiro de Saúde Mental, 1(1), $1-10$.

Mauer, S. K., \& Resnizky, S. (1987). Acompanhantes terapêuticos e pacientes psicóticos. Campinas, SP: Papirus.

Onocko-Campos, R. T., \& Furtado, J. P. (2006). Entre a saúde coletiva e a saúde mental: um instrumental metodológico para avaliação da rede de Centros de Atenção Psicossocial (CAPS) do Sistema Único de Saúde. Cadernos de Saúde Pública, 22(5), 1053-1062. doi: http://dx.doi.org/10.1590/ S0102-311X2006000500018.

Palombini, A. L. (2009). Utópicas cidades de nossas andanças: flânerie e amizade no acompanhamento terapêutico. Fractal Revista de Psicologia, 21(2), 295-317. doi: http://dx.doi. org/10.1590/S1984-02922009000200008

Pegoraro, R. F., \& Caldana, R. H. L. (2008). Sofrimento psíquico em familiares de usuários de um Centro de Atenção Psicossocial (CAPS) Interface - Comunicação, Saúde, Educação, 12(25), 295-307. doi: http://dx.doi.org/10.1590/ S1414-32832008000200006

Pitta, A. M. F. (2001). O que é reabilitação psicossocial no Brasil, hoje? In A. M. F. Pitta (Org.), Reabilitação psicossocial no Brasil (pp. 19-26, 2a ed.). São Paulo: Hucitec.

Pitiá, A. C., \& Furegato, A. R. (2009). O acompanhamento terapêutico (AT): dispositivo de atenção psicossocial em saúde mental. Interface - Comunicação, Saúde, Educação, 13(30), 67-77. doi: http://dx.doi.org/10.1590/ S1414-32832009000300007

Reis Neto, R., Teixeira Pinto, A., \& Oliveira, L. (2011). Acompanhamento terapêutico: história, clínica e saber. Psicologia: Ciência e Profissão, 31(1), 30-39. doi: http:// dx.doi.org/10.1590/S1414-98932011000100004

Sereno, D., \& Porto, M. (1991). Sobre acompanhamento terapêutico. In N. L. Carrozzo (Org.), A rua como espaço clínico (pp.41-50). São Paulo: Escuta.

Silva, M. V., Costa, F., \& Neves, L. (2010). Programa de intensificação de cuidados: experiência docente-assistencial em psicologia e reforma psiquiátrica. Psicologia: Ciência e Profissão, 30(4), 882-895. doi: http://dx.doi.org/10.1590/ S1414-98932010000400016

Venâncio, A. T. A. (2007). Sobre a desinstitucionalização psiquiátrica: história e perspectivas. História, Ciências, Saúde-Manguinhos, 14(4), 1415-1420. doi: http://dx.doi. org/10.1590/S0104-59702007000400017

Yasui, S., \& Costa-Rosa, A. (2008). A Estratégia Atenção Psicossocial: desafio na prática dos novos dispositivos de saúde mental. Saúde em Debate, 32(78-79-80), 27-37.

Zamignani, D. R., \& Wielenska, R. C. (1999). Redefinindo o papel do acompanhante terapêutico. In R. R. Kerbauy \& R. C. Wielenska (Orgs.), Sobre comportamento e cognição: psicologia comportamental e cognitiva - da reflexão teórica à diversidade na aplicação (pp. 156-163, Vol.4.) Santo André, SP: Esetec. 